\title{
Movimientos sociales en la Unidad Popular: reflexiones sobre ausencias y presencias teóricas
}

\author{
Francisco Javier Morales A. ${ }^{1}$ \\ Recibido: 25 de agosto de 2014 - Aceptado: 28 de septiembre de 2014
}

\begin{abstract}
Resumen
El estudio sobre los movimientos sociales y sus problemáticas más trascendentes parece haber tenido un desarrollo significativo en los últimos veinte años en Chile. Sin embargo, se trata de un proceso en permanente construcción, que requiere, por ende, de nuevas metodologías y nuevos marcos epistemológicos. En virtud de ello, ciertas temáticas parecen haber quedado relegadas a estudios o disciplinas muy específicas. Uno de estos tópicos se refiere a la ausencia -o presencia parcial- de lineamientos teóricos que acompañarán a los movimientos sociales durante la Unidad Popular. Este artículo propone una reflexión en torno a esta problemática, considerando sus alcances y coyunturas más significativas.
\end{abstract}

Palabras clave:Chile, Unidad Popular, movimientos sociales, teoría de la historia.

\section{Social movements during Unidad Popular: reflections on theoretical absence and presence}

\begin{abstract}
The study of social movements and their most significant issues has been an important subject for the last twenty years in Chile. However, it is a process in permanent construction which requires new methodologies and epistemological frameworks. In light of this, it seems that certain issues have been relegated to very specific studies and disciplines. One of these issues is related to the absence -or partial presence- of theoretical guidelines accompanying social movements during the Unidad Popular. This article proposes a reflection on this issue, considering its scope and most significant junctures.
\end{abstract}

Keywords: Chile, Unidad Popular, social movements, theory of history. 


\title{
Movimentos sociais na Unidade Popular: reflexões sobre ausências e presenças teoricas
}

\begin{abstract}
Resumo
0 estudo sobre movimentos sociais e suas problemáticas mais importantes parece ter tido um desenvolvimento significativo nos últimos vinte anos no Chile. No entanto, é um processo em permanente construção, exigindo, portanto, novas metodologias e novos enquadres epistemológicos. Em virtude disso, certos temas parecem ter sido relegados para estudos ou disciplinas muito específicas. Um desses tópicos refere-se à ausência -ou presença parcial- das orientações teóricas que acompanham aos movimentos sociais durante a Unidade Popular. Este artigo propõe uma reflexão sobre esta questão, considerando sua importância e conjuntura mais significativa.
\end{abstract}

Palavras-chave: Chile, Unidade Popular, movimentos sociais, teoria da história.

La Unidad Popular no sólo fue la culminación del largo proyecto histórico que la izquierda chilena construyó en el transcurso del siglo XX, sino también una etapa en que los más diversos movimientos sociales lograron desplegarse a plenitud. La discusión y acción en el Chile unipopular rebasaría con creces los márgenes de lo político e institucional. Cada espacio o dinámica que se articuló por aquel entonces parecía estar condicionada por una atmósfera de cambio y transformación, de ruptura y avance.

Los prácticamente tres años de la llamada Vía Chilena al Socialismo despertaron desde un comienzo el interés de la comunidad regional e internacional. Como nunca antes, al decir de algunos autores, Chile se vinculó y dialogó con el mundo entero, en un esfuerzo no menor, dada la particularidad del experimento chileno (Fermandois, 2013).

El interés por lo que sucedería en Chile no sólo se expresó en el campo de las relaciones internacionales, pues las Ciencias Sociales rápidamente tomaron nota del significado y trascendencia que un proyecto de esta envergadura significaba para el país y la región. Buena parte de las investigaciones que buscaron retratar los años de la Unidad Popular parecen compartir al menos dos énfasis bien característicos; en primer lugar, describen una trayectoria esencialmente política e institucional, detallando con pulcritud las dinámicas que envolvieron a los principales partidos y organizaciones del período. En segundo término, estos estudios se encuentran indisolublemente ligados al trágico final que tuvo el gobierno allendista, sugiriendo de paso que la derrota del proyecto socialista se debió en gran medida a 
tensiones y fracturas en el sistema institucional. ${ }^{2}$ En otras palabras, lo político, en su acepción más tradicional, determinó la forma como se analizó, desde la Academia, la experiencia chilena.

Recién en los últimos veinte años han surgido nuevas perspectivas de análisis sobre la Unidad Popular, en donde el foco de atención no se centra únicamente en lo político sino que considera nuevas dinámicas, nuevos actores y nuevas metodologías (Del Pozo, 1992; Rolle -coord.-, 2003; Winn, 2004; Garcés, 2005; Tinsman, 2009). La importancia de estas investigaciones radica en que además de abrir el abanico temático, invitan a repensar desde otras ópticas la experiencia socialista en Chile.

En este sentido, la Unidad Popular fue bastante más que una alianza de partidos políticos. Junto al plano institucional, existió también un espacio temporal y factual en donde intervinieron trabajadores, pobladores, campesinos, mujeres y jóvenes quienes dieron vida a un amplio mosaico de experiencias y realidades que como nunca antes parecieron eclosionar en Chile.

Se trató, en definitiva, de aquellos movimientos sociales que fueron los actores principales de la Vía Chilena al Socialismo, ya sea por sus dinámicas y trayectorias -que les fueron inherentes a su condición de tales- pero también porque la propia Unidad Popular, sin conceptualizar acabadamente esta idea, los visualizaba como parte integrante de su proyecto estructural. Pensamos específicamente en movimientos como el de los pobladores, los trabajadores agrupados en torno a los cordones industriales y otros.

A la luz de tales consideraciones, este artículo busca reflexionar, de forma muy preliminar por cierto, sobre la relación existente entre teoría y movimientos sociales durante la Unidad Popular. Dicho propósito parte del supuesto de que la teoría sobre movimientos sociales es algo relativamente nuevo dentro de las Ciencias Sociales en general, por lo que su corpus epistemológico se encuentra en permanente construcción y avance.

Las reflexiones que aquí se proponen buscan preguntarse por las razones que explican una ausencia de teoría sobre movimientos sociales durante la Unidad Popular, y más específicamente sobre si dicha ausencia fue un factor relevante para la viabilidad y proyección de dichos movimientos. En una

2 La bibliografía sobre la experiencia chilena de la UP resulta realmente gigantesca. Se puede, sin embargo, clasificar ciertos textos de acuerdo a su orientación y contenidos más significativos. De este modo, los principales estudios que responden a la perspectiva institucional y política del asunto son los siguientes: Angell (1993); Corvalán (2004); y Valenzuela (1978). Existen igualmente algunos estudios que desarrollan una reflexión desde la perspectiva de los protagonistas del proceso político vivido bajo la UP, como es el caso de Bitar (1979); Garcés (1972) y Arriagada (1974). 
segunda parte, se trazará un análisis sobre dos de los movimientos sociales más representativos durante la Unidad Popular; el movimiento de pobladores y los denominados cordones industriales.

\section{Interrogantes teóricas sobre los movimientos sociales durante la Unidad Popular}

Diversos cientistas sociales han hecho saber lo paradójico que resultó para la Unidad Popular llevar adelante una revolución social y política careciendo de una teoría que pudiera conducir y sustentar la movilización que inevitablemente acompañaría el proceso de cambios. Tomás Moulian (2005: 37), es enfático al sostener lo problemático que resultaba conducir un proceso de las dimensiones de la Unidad Popular bajo el silencio y la improvisación de una teoría marxista que no había reflexionado ni construido las herramientas necesarias para una experiencia de este tipo. Incluso más, Salazar (2012: 436) señala que el término movimiento social aparece por primera vez en la década del cuarenta en Chile, para reaparecer sólo bajo el periodo dictatorial.

Esta ausencia de teoría social, en todo caso, no es algo de lo que deba responsabilizarse únicamente a la Unidad Popular y sus ideólogos. El vacío conceptual a este respecto parecía ser transversal a las disciplinas académicas en general. Cabe precisar además, que lo que se entendía por movimiento social en los sesenta y setenta -aun cuando no se utilizara específicamente ese término- dista mucho de las elaboraciones conceptuales que a este respecto han surgido en los últimos años.

La clásica historiografía marxista chilena (Ramírez, 1956, y Barría, 1971), consideró -sin explicitar conceptualmente esta idea- que lo social era sinónimo de movimiento obrero. Sin hacer mayores construcciones conceptuales, la historia de los trabajadores en Chile, según esta visión, parecía tener un origen claro y una trayectoria bastante nítida. Así, el movimiento obrero tuvo su origen en la pampa salitrera del Norte Grande, desde donde se expandiría al resto del país. En dicho proceso, el rol de los partidos de clase resultaría clave para viabilizar y conducir con éxito, a lo largo del siglo XX, al movimiento obrero. Se trata de una interpretación extremadamente rígida, acaso mecánica, pero que, sin embargo, pareció nutrir política e ideológicamente a segmentos importantes del proletariado nacional.

En los últimos años, afortunadamente, han surgido investigaciones que ponen en entredicho esta interpretación, logrando rescatar una serie de procesos y actores que hasta ahora parecían olvidados o derechamente ignorados. Tal fue el caso del papel jugado por el anarco-sindicalismo en las primeras décadas del siglo pasado y que De Shazo (2007) reconstruye brillantemente. 
La carencia entonces de trabajos teóricos que analizaran a los movimientos sociales durante la UP se explicaría en parte porque los lineamientos epistemológicos que predominaban por aquel entonces no lograron visualizar a aquellos actores que provenían de realidades ajenas al mundo estrictamente obrero y, además, porque las propias disciplinas humanísticas parecían haber "olvidado" el significado y proyección del término movimiento social. Sólo a partir de los cambios que supuso el tránsito de una sociedad industrial a una postindustrial, fue posible advertir la presencia y dinámicas de otros actores sociales. Las disciplinas académicas, al tomar nota de estos nuevos protagonistas, procedieron rápidamente a conceptualizar sus trayectorias y proyecciones bajo el rótulo de Nuevos Movimientos Sociales.

La Unidad Popular, en todo caso, se sitúa contextualmente bajo el paraguas de la sociedad industrial, por lo que no debe extrañar ni el protagonismo del movimiento obrero ni la ausencia de herramientas teóricas que condujeran la acción social. Dicha ausencia, en todo caso, no fue un problema que afectase únicamente a la Unidad Popular. Otras épocas y experiencias, muy activas en cuanto a la movilización social, también carecieron de dichas herramientas. Ciertamente, el contexto era diferente y sus protagonistas - por ejemplo, en el contexto del ciclo huelguístico de comienzos del siglo XX- se encontraban en una posición esencialmente subordinada con respecto al poder político-institucional. Probablemente, sus luchas y demandas no permitían visualizar la ausencia de una teoría social que respaldase su accionar.

Bajo el gobierno de Allende, en cambio, las fuerzas sociales y populares alcanzaron una posición inmejorable para concretar sus anhelos y esperanzas de cambio. No se trataba, como antaño, de interpelar simplemente al Estado en busca de mejores condiciones de vida, sino que existía la posibilidad de transformar política y económicamente al país mediante una revolución socialista, la cual en todo caso sería dirigida desde arriba y bajo el control de los partidos y sus funcionarios.

La forma en que se llevaría a cabo dicho proceso, o más bien, los instrumentos prácticos y teóricos que conducirían a los sectores sociales que apoyarían a la UP, parecen ser uno de los puntos más inquietantes para algunos cientistas sociales. La pregunta central a este respecto parece ser la siguiente: ¿cómo un proyecto de la envergadura y significado histórico de la Unidad Popular careció de la teoría que permitiera conducir conceptualmente la movilización social que le acompañaría? Desde dicha interrogante, se deriva también la pregunta sobre el modo en que la izquierda construyó teóricamente su proyecto o, más específicamente, ¿por qué si dicha construcción se fue haciendo a lo largo del siglo XX, no se pensó y reflexionó acabadamente sobre los actores que sustentarían la revolución socialista? 
Estas reflexiones, en todo caso, abren el espacio para una interrogante mayor, igualmente importante a nuestro entender. Esta se refiere a la funcionalidad que una teoría sobre movimientos sociales -en caso de haber existido- hubiese significado para la Unidad Popular. En otras palabras, la pregunta es acerca de la utilidad que habría tenido, para los actores en su conjunto, un cuerpo teórico que ayudara a conducir el proceso político y social durante la UP. En forma paralela, cabe preguntarse sobre cómo se instrumentalizaría y pondría en práctica dicha teoría, o mejor dicho; ¿en qué momento se produciría el ejercicio intelectual de conocer y asimilar la teoría -por parte de los movimientos sociales- a fin de ponerla en práctica y hacerla parte integrante de sus estrategias más inmediatas?

No resulta fácil responder de forma acabada a tales interrogantes. Más bien, ellas abren un espacio para reflexionar sobre los alcances y problemas que se generan a partir de la relación entre teoría, movimientos y acción social.

A este respecto, el proceso vivido en el Chile de la Unidad Popular tuvo como característica esencial la diversidad de formas y estrategias que los actores sociales construyeron a lo largo del período. No se trató de una experiencia compacta y homogénea, en donde las bases simplemente asintieran a las determinaciones adoptadas por la dirigencia. Cada movimiento social visualizó y asimiló desde su propia realidad y entorno el proceso de cambios que se iniciaba en Chile.

Así, no fueron simplemente los partidos políticos y sus militantes quienes sustentaron al régimen de Allende. También hubo una diversidad de movimientos sociales que se sintieron parte integrante del gobierno popular. Más aún, dichos movimientos elaboraron, al contrario de las lógicas partidistas -siempre tan institucionalizadas y formales-, sus propias estrategias y cursos de acción. Bajo ese escenario, los constructos teóricos que pudieran haber existido tendrían que haber hecho un esfuerzo no menor por insertarse y legitimarse al interior de la base social.

Existe otro elemento que también pone en entredicho las verdaderas capacidades y contribuciones que una teoría sobre movimientos sociales habría tenido para el gobierno socialista. Este se refiere al espacio temporal en que los movimientos sociales habrían pensado y aquilatado la teoría social a fin de hacerla parte integrante de sus estrategias y cursos de acción. Si consideramos lo complejo que fue la implementación del programa de la UP, tanto por la oposición que inevitablemente surgiría, como también por la improvisación con que se buscaba materializarlo, las posibilidades de pensar conceptualmente el proceso se hacían escasas. Los hechos parecían desbordar periódicamente la realidad y, por ende, la capacidad de acción y control de sus propios protagonistas. 
En virtud de lo anterior, los diagnósticos y reflexiones partidistas evidenciaron énfasis esencialmente coyunturales, influenciados por el calor de la contingencia y subsidiarios, en gran medida, de herramientas ideológicas muy básicas. Esta situación, en vez de contribuir en el desarrollo de diagnósticos consistentes, más bien deformaba el alcance y proyección material de los problemas. Cabe puntualizar, por cierto, que este no fue un fenómeno exclusivo de la izquierda, pues en términos generales todos los actores políticos adolecieron de esta contrariedad. En muchos casos, los diagnósticos y análisis fueron extremadamente exagerados. Ni el gobierno se encaminaba a convertirse en un Estado totalitario del tipo soviético, como acusaba la derecha, ni tampoco la experiencia chilena -según ha apuntado acertadamente Moulian (2005: 38)- podía homologarse a la situación vivida en Rusia en 1917, pues el Estado no se estaba "cayendo a pedazos". Sin embargo, y en la medida en que tales diagnósticos se agudizaran y más aún constituyeran el punto de partida para analizar la contingencia, menos espacio habría para legitimar un trabajo teórico que condujera la movilización social.

Bajo ese esquema, las consecuencias prácticas de un trabajo conceptual que guiara, o al menos analizara, a los movimientos sociales durante la UP habrían sido más bien mínimas. Sobre todo porque dichos movimientos se encontraban elaborando sus propios cursos de acción, es decir, estaban experimentando su propia revolución, a la cual, dicho sea de paso, llegaron después de un largo periodo de luchas y esfuerzos mancomunados. En todo este proceso de autoconstrucción de un proyecto sectorial y social, la teoría fue posiblemente un factor muy poco relevante.

\section{Movimientos sociales durante la UP: una mirada teórica e histórica desde el presente}

Considerando las reflexiones hasta aquí desarrolladas, cabe preguntarse por las posibilidades de analizar teóricamente a algunos de los movimientos sociales que actuaron bajo la Unidad Popular. Este ejercicio supone pensar a dichos movimientos desde el presente y a partir de categorías elaboradas en los últimos veinticinco años, situación que permitirá una comprensión mayor de su accionar colectivo a partir de las estrategias que lograron desarrollar.

Preguntarse por los rasgos que definen a un movimiento social ha sido una interrogante que se han planteado diversos cientistas sociales a lo largo del tiempo. Quizás con mayor detención en los últimos 30 años, gracias a las innumerables experiencias colectivas que eclosionaron en distintas partes del mundo, pero también por la visibilidad que estas experiencias tuvieron en un contexto de amplia difusión mediática. 
La diversidad de respuestas que intentaron hacer frente a la pregunta más arriba indicada, y que además realizaron aportes complementarios al fenómeno de los movimientos sociales, posee una trayectoria conceptual y académica fuertemente vinculada al mundo anglosajón. Ha sido la sociología norteamericana y europea la que más aportes ha realizado a este respecto. Una buena síntesis de estos recorridos teóricos se puede encontrar en Mc Adam, Mc Carthy y Zald (1996); Melucci (1999) y Della Porta y Diani (2011).

En el trabajo de Della Porta y Diani se desarrolla un acertado análisis sobre aquellos elementos que dan forma y perfilan a un movimiento social. Conviene por lo mismo tomar nota de dichos elementos a fin de contrastar su contenido con las trayectorias y experiencias de algunos movimientos sociales que actuaron durante la UP.

El primer elemento explicitado por estos autores se expresa a través de una premisa básica, a saber, que los movimientos sociales son esencialmente procesos sociales diferenciados, esto quiere decir que no cualquier acción colectiva llega a constituirse como un movimiento social (2011: 43). Se debe distinguir por lo mismo un movimiento relativamente cohesionado y con objetivos específicos de aquellas acciones espontáneas que sólo manifiestan un descontento muy acotado y coyuntural. Al mismo tiempo, Della Porta y Diani señalan que los procesos sociales presentan una serie de mecanismos a través de los cuales los actores se encuentran comprometidos en la acción colectiva mediante tres elementos; primero, su participación en relaciones conflictivas con oponentes claramente diferenciados; segundo, su vinculación a densas redes de carácter informal y, tercero, el compartir una identidad colectiva diferenciada (2011: 43-45). ¿Fueron distintivos estos rasgos en algunos movimientos sociales durante la UP?

Posiblemente sí, aunque siempre caben matices que complejizan y ensanchan la reconstrucción y el análisis histórico. El primer elemento -la participación en relaciones conflictivas con oponentes diferenciados- parece explicitarse por sí mismo. El movimiento de pobladores, los trabajadores que fueron parte de los denominados cordones industriales y otro tipo de movimientos bien parecen evidenciar este tipo de comportamientos.

En el caso de los pobladores, cabe precisar que su organización no se inició con la UP, pues su desarrollo se venía gestando, según ha retratado Garcés (2002; 2011), desde varias décadas atrás. Dicho movimiento fue organizándose a través de diversas estructuras y prácticas que buscaban concretar sus demandas habitacionales. Bajo la UP, el avance de los movimientos de pobladores siguió una línea ascendente con respecto al período anterior expandiendo como nunca antes las tomas de terrenos. Este tipo de acciones, derivaría en muchos casos en la constitución de innumerables poblaciones en casi todo Santiago. 
El cuadro parece complejizarse, en todo caso, si tomamos en cuenta que las principales demandas de este movimiento se hicieron en dirección al Estado, y que cuando éste no fue capaz de reaccionar ni responder a aquellas, los pobladores desarrollaron sus propios mecanismos y prácticas para solucionar dichos problemas. En cierto modo, el principal oponente fue el Estado, considerando sus respuestas tradicionalmente tardías y parciales. Sin embargo, la llegada de la UP al gobierno supuso una dinamización de las políticas con que el Estado había hecho frente al problema habitacional, de modo que éste evolucionaría hacia una posición de mediación y concreción de las demandas del mundo poblacional.

Las redes informales de las que hablan Della Porta y Diani fueron a todas luces una característica esencial del movimiento de pobladores durante la UP, aunque cabe insistir en que dichas redes se articularon con anterioridad a la llegada de la izquierda al poder. Desde prácticamente mediados de los cincuenta, los pobladores desarrollaron prácticas e intercambios de recursos en la perspectiva de concretar metas y anhelos que involucraron a sectores bastante diferenciados y que además no perdieron su autonomía ni su independencia. En este sentido, la identidad colectiva que nutrió el accionar del movimiento de pobladores sería igualmente un elemento clave, puesto que otorgaba un sentido de pertenencia y sociabilidad que trascendía lo individual. Antes de identificarse políticamente como sujetos de izquierda, muchos actores se sentían pobladores. Era en el espacio territorial de la toma o la población en donde se desarrollaba la experiencia social y colectiva, y en donde se construía una identidad común y diferenciada.

El caso de los cordones industriales adquirió una importancia decisiva en la UP luego del denominado Paro de octubre de 1972. Los trabajos historiográficos más representativos a este respecto, dada su profundidad y análisis, corresponden a los de Cancino (1988) y Gaudichaud (2004).

Reconstruyendo algunos hitos de esta coyuntura, cabe señalar que la paralización de octubre fue promovida desde el gremio transportista, a propósito de un intento de estatización de una empresa de camiones ubicada en la región de Aysén. Con el paso de las semanas, otros gremios empresariales se sumaron al paro en lo que pareció ser una maniobra conjunta a fin de desestabilizar al gobierno y lograr finalmente su caída.

En ese contexto, los sectores proletarios comprometidos con el gobierno de Allende desarrollaron una serie de iniciativas tendientes a ocupar fábricas e industrias a fin de mantener, en la medida de lo posible, el ritmo productivo del país. Estas organizaciones, denominadas igualmente como "comandos comunales" o "comandos coordinadores", lograron complementarse con otro tipo de organizaciones como las Juntas de Abastecimiento y Precios (JAP) o algunas Brigadas de Vigilancia con el objetivo 
de llevar a cabo una distribución relativamente eficiente de mercaderías y productos que para ese entonces, y a consecuencia del lock out de octubre y de la expansión del mercado negro, escaseaban en sectores importantes de la población.

En cuanto a su significado y trascendencia, los cordones industriales lograron dinamizar la movilización popular además de descentralizar la actividad política y replantear abiertamente el tema de las relaciones de producción, según lo ha comentado acertadamente Franck Gaudichaud (2005: 95). Se trató en definitiva de organizaciones horizontales, con dinámicas e identidades propias, pero que además lograron movilizar una cantidad no menor de recursos en el logro de sus objetivos más inmediatos.

Según el esquema conceptual propuesto por Della Porta y Diani, estas organizaciones cumplieron a cabalidad con los elementos que distinguen a un movimiento social. En primer término, desarrollaron relaciones conflictivas en virtud de oponentes claramente identificados, en este caso los gremios empresariales. Seguidamente, lograron articular densas redes informales a fin de conducir sus estrategias de mediano y largo plazo y alcanzar finalmente sus objetivos. La concreción de estas redes, que incluyeron, por ejemplo, a algunos camioneros que no se plegaron al paro, logró aminorar en buena medida los efectos materiales de una larga paralización. De igual modo, la identidad colectiva que nutrió el accionar de los cordones industriales perfiló experiencias únicas en cuanto al reconocimiento y pertenencia a organizaciones que compartían un diagnóstico común en relación a la crítica situación por la que atravesaba el país. Los nombres que adoptaron los propios cordones industriales (Vicuña Mackenna, Cerrillos, O'Higgins, etc.) reflejaron en parte esa pertenencia e identidad colectiva que asumieron sus integrantes.

Pero los efectos materiales del Paro de octubre implicaron también un cuestionamiento a la forma como se miraba y proyectaba la Vía Chilena al Socialismo en su conjunto, y más específicamente cómo se definiría el tema del poder. A partir del surgimiento de los cordones industriales, pero también por las elaboraciones político-estratégicas que un sector de la izquierda hiciese al respecto, el término poder popular pareció alcanzar cierto grado de materialización.

En la práctica, esto suponía cuestionamientos no menores a la estrategia gradualista que defendía un sector de la izquierda, incluido el propio Presidente Allende, puesto que pasaba por alto los mecanismos esencialmente institucionales que debían articular la puesta en marcha del programa de gobierno. Desde otra perspectiva se trataba de un problema conceptual, e inclusive ideológico, pues significaba dilucidar si la experiencia unipopular constituía un intento por alcanzar el socialismo desde bases institucionales, o 
bien, si a raíz de la acción mancomunada de la oposición -tendiente a derrocar al gobierno- la respuesta del gobierno sería apostar por la contraofensiva de los movimientos sociales.

\section{Reflexiones finales}

En las páginas precedentes, se plantearon ciertas interrogantes sobre la relación entre teoría y movimientos sociales durante la Unidad Popular. Como punto de partida, se explicitaron una serie de preguntas sobre la ausencia, o eventual presencia, de un cuerpo teórico sobre movimientos sociales durante la UP.

A decir verdad, dicha ausencia no implicaba una cuestión únicamente conceptual o académica, pues implícitamente posee un trasfondo político. En este trabajo se desarrollaron al menos dos elementos que intentan complementar el tema teórico. En primer lugar, y suponiendo que sí hubiese existido un cuerpo conceptual sobre movimientos sociales durante la UP, la pregunta que cabe enunciar es acerca de su utilidad práctica a la hora de conducir un proyecto de carácter estructural que apuntaba, como sabemos, a transformar radicalmente las relaciones sociales y económicas del país, y que en la práctica se vio constreñido y condicionado por la contingencia política.

En segundo término, se explicitó una pregunta sobre los espacios y momentos en que las organizaciones sociales pensarían la teoría. Sabemos que el tempus social es distinto del tiempo institucional, por lo que los ritmos y estrategias de uno y otro sector raramente son coincidentes. Podríamos apuntar también que la teoría se construye esencialmente desde la academia y desde allí se expande hacia el cuerpo social, pero ello no necesariamente implica que las organizaciones asimilen y hagan suya esa teoría. En la base, los movimientos sociales construyen experiencia y, sobre todo, acción colectiva.

Cabe precisar además que los problemas derivados de una ausencia de herramientas teóricas no fueron factores que impactaran únicamente en el accionar de los movimientos sociales durante la UP. Visto desde un plano político-estratégico, el propio gobierno se encontraría huérfano de algún paradigma o modelo que le permitiera hacer frente a una realidad que pareció desbordarlo periódicamente. Aun cuando existieran algunas herramientas o rutas de navegación para conducir inicialmente el proceso, estas se mostrarían, según Moulian, como insuficientes, pues la gran discusión teórica que ameritaba un proceso como la UP nunca se realizó (2005: 37).

Gracias a los constructos teóricos que se han desarrollado en los últimos años, ha sido posible reconocer aquellos elementos distintivos de los mo- 
vimientos sociales y populares que dinamizaron el cuadro político durante la UP. Así, este trabajo centró su mirada en el movimiento de pobladores y en el surgimiento de los denominados cordones industriales. El primero de ellos parece no haber sido aquilatado por la dirigencia de la UP, ni política ni estratégicamente, subvalorando su capacidad de organización y proyección política. Los cordones industriales, en tanto, sí despertaron cierto interés en la dirigencia oficialista. A decir verdad, el protagonismo alcanzado por los cordones tras el paro patronal de octubre de 1972, supuso una discusión política no menor en el seno de la UP. El dilema fundamental que enfrentó Allende y los partidos por aquel entonces se refería a si la Vía Chilena al Socialismo debía apoyarse en la acción y dinamismo de los sectores proletarios, el denominado poder popular, o bien, conducir el proceso desde el aparato institucional y de forma gradual. Estas discusiones, claro está, se hicieron en condiciones conceptualmente precarias, en donde los diagnósticos y cursos de acción se veían mediatizados y condicionados por la contingencia. En ese marco, resultaba muy difícil proyectar soluciones estratégicas de largo plazo, que permitieran, en fin, viabilizar el programa de transformación estructural de la UP.

Nuestra perspectiva de análisis, en el caso del movimiento de pobladores y de los cordones industriales, apuntó a develar trayectorias y experiencias que sólo en los últimos años, y en parte gracias al aporte de la teoría social, parecen haber ingresado en los estudios historiográficos.

\section{Referencias bibliográficas}

\section{Artículos}

GARCÉS, M. (2005). "Construyendo 'las poblaciones'. El movimiento de pobladores durante la Unidad Popular", en Pinto, J., ed. (2005), Cuando hicimos historia. La experiencia de la Unidad Popular. Santiago: LOM, p. 57-79.

GARCÉS, M. (2011). "Los pobladores durante la Unidad Popular: Movilizaciones, oportunidades políticas y la organización de las nuevas poblaciones", en Tiempo Histórico, №3, p. 37-53.

GAUDICHAUD, F. (2005). "Construyendo 'Poder Popular': El movimiento sindical, la CUT y las luchas obreras en el periodo de la Unidad Popular, en Pinto, J., ed. (2005), Cuando hicimos historia. La experiencia de la Unidad Popular. Santiago: LOM, p. 81-105.

MOULIAN, T. (2005). "La vía chilena al socialismo: Itinerario de la crisis de los discursos estratégicos de la Unidad Popular", en Pinto, J., ed. (2005), Cuando hicimos historia. La experiencia de la Unidad Popular. Santiago: LOM, p. 35-56. 


\section{Libros}

ANGELL, A. (1993). Chile de Alessandri a Pinochet, en busca de la utopía. Santiago: Andrés Bello.

ARRIAGADA, G. (1974). De la vía chilena a la vía insurreccional. Santiago: Editorial del Pacífico.

BARRÍA, J. (1971). El movimiento obrero en Chile. Santiago.

BITAR, S. (1979). Transición, socialismo y democracia: La experiencia chilena. México: Siglo XXI.

CANCINO, H. (1988). La problemática del poder popular en el proceso de la vía chilena al socialismo, 1970-1973. Dinamarca: Aarhus University Press.

CORVALÁN, L. (2004). Los partidos políticos y el golpe del 11 de septiembre. Santiago: Universidad Bolivariana.

DELLA PORTA, D. y M. Diani (2011). Los movimientos sociales. Madrid: Ed. Universidad Complutense de Madrid.

DEL POZO, J. (1992). Rebeldes, reformistas y revolucionarios: una historia oral de la izquierda chilena en la época de la Unidad Popular. Santiago: Documentas.

DE SHAZO, P. (2007). Trabajadores urbanos y sindicatos en Chile, 1920-1927. Santiago: DIBAM.

FERMANDOIS, J. (2013). La revolución inconclusa. La izquierda chilena y el gobierno de la Unidad Popular. Santiago: Centro de Estudios Públicos.

GARCÉS, J. (1972). Chile: el camino político hacia el socialismo. Barcelona: Ariel.

GARCÉS, M. (2002). Tomando su sitio. El movimiento de pobladores en Santiago, 1957-1970, Santiago: LOM.

GAUDICHAUD, F. (2004). Poder popular y cordones industriales. Testimonios sobre el movimiento popular urbano, 1970-1973. Santiago: LOM.

MC ADAM, D., J. Mc Carthy, y M. Zald. (1996). Movimientos Sociales. Perspectivas comparadas. Oportunidades políticas, estructuras de movilización y marcos interpretativos culturales. Madrid: Istmo.

MELUCCl, A. (1999). Acción colectiva, vida cotidiana y democracia. México: El Colegio de México.

RAMÍREZ N., H. (1956). Historia del Movimiento Obrero en Chile. Santiago: Lautaro.

ROLLE, C. (Coordinador) (2003). 1973. La vida cotidiana de un año crucial. Santiago: Planeta. 
SALAZAR, G. (2012). Movimientos sociales en Chile. Trayectoria histórica y proyección política. Santiago: Uqbar.

TINSMAN, H. (2009). La tierra para el que la trabaja. Género, sexualidad y movimientos campesinos en la Reforma Agraria chilena. Santiago: LOM-Centro de Investigaciones Diego Barros Arana.

VALENZUELA, A. (1978). El quiebre de la democracia en Chile. Santiago: FLACSO.

WINN, P. (2004). Tejedores de la Revolución. Los trabajadores de Yarur y la vía chilena al socialismo. Santiago: LOM. 